\title{
Reviewer comment
}

a) There is a big confounder of having alternative care personal in the family, as this will affect both individual as well as relationship with partner and until multivariate regression looks into this the causality cannot be established

b) The LISS data needs to explained as there can be bias due to the dataset itself

c) Difference of pre and post covid is not being established through background as well as result section, some relationship demonstration will be extremely important. 